\title{
Texturization of Engine Components with Shaped Ultrashort Laser Pulses
}

\author{
Wagner de Rossi ${ }^{1}$ and Alexandre Vieira ${ }^{2}$ \\ ${ }^{1}$ Centro de Lasers e Aplicações - IPEN-CNEN/SP - Av. Prof. Lineu Prestes, 2242, 05508-000, São \\ Paulo, Brazil \\ E-mail:wderossi@gmail.com \\ ${ }^{2}$ Faculdade de Tecnologia SENAI Suiço-Brasileira "Paulo Ernesto Tolle”, St. Bento Branco de \\ Andrade Filho, 379, 04757-000, São Paulo, Brazil
}

\begin{abstract}
The aim of this work was to texturize surfaces of steel engine components to reduce the coefficient of dynamic friction and wear between two surfaces. The elements of the texture had the shape of a spherical half-shell and were produced by the overlap of femtosecond laser pulses with donut shape. The incubation effect was estimated using the D-scan method to relate the ablation threshold to the number of overlapping pulses. Thus, the control of the focal position of the laser beam and the number of overlapped pulses, taking into account the effects of incubation, made it possible to obtain this type of dimple with controlled shape and size. A distribution of dimples with density of $11 \%$ caused an $18 \%$ reduction in the coefficient of friction in a tribological pair DIN $16 \mathrm{MnCr} 5$ and SAE 52100 steel sphere.
\end{abstract}

DOI: $10.2961 /$ jlmn.2019.02.0005

Keywords: micromachining, femtosecond laser, shaped laser beam, laser texturing, friction force.

\section{Introduction}

Micromachining is an important field of study for many knowledge areas, however, the most of applications are concentered in aerospace, microelectronics, automotive, optics, medical and odontology, in manufacturing of some devices like micro electrical mechanical systems (MEMS), microfluidics chips, micromotors, and others [1]. Due to these increasing applications, it is necessary to develop applied researches related of this field.

In automotive industry, one of the promising applications of micromachining techniques is the laser surface texturing (LST), mainly to reduce the coefficient of friction and reduce the effects of wear, which improve the lifetime of the engine components $[2,3,4,5,6]$. Usually nanosecond laser pulses are used in LST processes; this however gives rise to some drawbacks especially harmful to engine components like heat affected zone (HAZ), micro cracks, remelted material, debris and low geometry control $[7,8]$.

To prevent or reduce such effects, mainly in metallic materials, the use of ultrashort laser pulses is becoming an effective alternative. Although it does not prevent the production of heat in metals, the thermal effects of ultrashort pulses in the vicinity of the affected region are very difficult to detect and can be neglected in most practical applications [9].

In internal combustion engines, the friction effects have an important negative influence, for instance, piston system generates around $1 / 3$ of loss of energy, being that, $11 \%$ of the total energy is used to overcome the friction influences. In this scene, $5 \%$ of reduction of friction lost in the engines, represents a total reduction in the annual consumption of fuel around 117 billion of liters, corresponding to a reduction of 290 billion tons of $\mathrm{CO}_{2}$ in the atmosphere [10].

The most common microstructures used in such textures are semi-spherical micro cavities following different spatial distributions that can vary in their design, alignment and tex- tured area. These microstructures can assume different functions depending on the application and characteristics of the systems. They can act as micro bearings when the texture gives rise to a flat surface due to the hydrodynamics pressure of the oil inserted into the dimple [11]. When the dimensions of the structures are bigger, the hydrodynamics effects no longer occur, and they assume the role of a lubricant reservoir, improving the conditions of critical lubrication. When detached particles are adhered on the surface, these microstructures can also behave like a debris trapper and a hard particle breaker.

\section{Experimental}

This works shows the developments to produce a texturized surface in DIN $16 \mathrm{MnCr} 5$ steel, widely used in combustion engine components, using ultrashort laser pulses. It was used a Ti:sapphire amplified laser system from Femtolasers with polarized emission centered at $800 \mathrm{~nm}$, pulselength of $30 \mathrm{fs}$, maximum energy of $200 \mu \mathrm{J}$ and $10 \mathrm{kHz}$ of repetition rate. The laser beam was focused by a doublet lens $40-\mathrm{mm}$ focal length that produces a focal spot of $4.8 \mu \mathrm{m}$ in diameter. The sample was moved by a 3D stage programmed by dedicated cadcam software.

The microstructure chosen here was that of semi-spherical micro cavities hereafter referred to simply as dimples. Their ideal diameter sought were in the range between 40 and $100 \mu \mathrm{m}$ keeping a ratio of $10 / 1$ to depth.

\subsection{D-scan Technique}

The D-Scan technique [12] was used to determine the ablation threshold of the material as function of the number of overlapping pulses. The D-Scan traces were also used to produce different morphologies formed by the use of different energy densities and number of overlapped pulses. In this technique, the focal spot traverses the sample from a point 
within the material to a point above the surface. Simultaneously, it is moved perpendicular to the propagation direction of the beam. Usually, both movements are made at the same speed. The result of this type of beam scan is the production of a groove like those shown in figure 1. In [12] it was shown that ablation threshold $F_{\text {th }}$ can be obtained by a simple relationship between the energy $\mathrm{E}_{0}$ used and the maximum width of the scratch. This relationship is given by:

$$
F_{t h}=\frac{E_{0}}{e \pi \rho_{\max }^{2}} \approx 0.117 \frac{E_{0}}{\rho_{\max }^{2}}
$$

where $\rho_{\max }$ is half of the ablation profile maximum transversal dimension; " $\mathrm{e}$ " is the Euler's number $(\mathrm{e} \sim 2.71828)$ and " $\mathrm{f}$ " is the used repetition rate. In addition, under these conditions, the number $\mathrm{N}$ of overlapping pulses is given by:

$$
N=1.8 \frac{f \rho_{\max }}{v_{y}}
$$

where $v_{\mathrm{y}}$ is the transversal translation speed.

Many traces can be produced with different ratios of repetition rate and displacement speeds corresponding to different number $\mathrm{N}$ of pulses overlap. In this way, a large amount of data can be obtained and the behavior of $F_{\text {th }}$ as a function of $\mathrm{N}$ can be readily obtained.

\subsection{Tribology tests}

Tribological tests were done in textured surfaces with a tribometer TE-67 Plint\&Partners Ltda. The test setup chosen to study the texture behavior in critical conditions of contact was sphere-on-disc lubricated, and all test proceedings followed the ASTM G99 recommendations. An external peristaltic pump was used to provide lubrication; the selected lubricating oil was a commercial synthetic SAE 5W30 (ILSAC GF-5 Lubrax Petrobras). This choice took into account the high use of this lubricant in vehicles produced in Brazil with flex-fuel combustive (gasoline and ethanol).

The test setup parameters were keep constant in all tests. The used speed was $1 \mathrm{~m} / \mathrm{s}$ and axial force of $30 \mathrm{~N}$, controlled by a load cell (Phoenix Tribology) and a pneumatic valve; the system guarantees an accuracy of $\pm 0,2 \mathrm{~N}$. A sphere of SAE 52100 steel was used as counter-body. The total length of each test was $1 \mathrm{~km}$.

The disc surface was grinded up to a roughness of $\mathrm{Ra} 0.3$ $\mu \mathrm{m}$, and tests were performed with cemented discs (54 HRC).

\section{Results}

Figure 1 shows the image of several D-Scan traces with

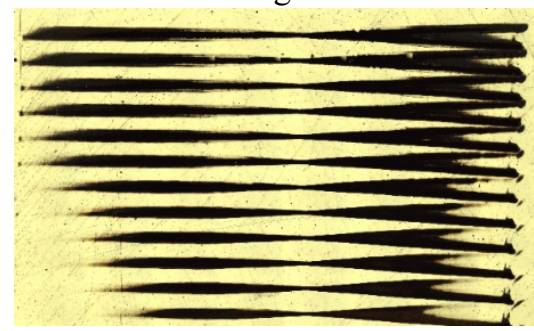

Fig. 1 D-Scan marks observed by confocal microscopy. Different scratches were produced with different Ns; from $\mathrm{N}=8$ to $\mathrm{N}=2,140$ from down to top. different Ns obtained by confocal microscopy. The images were used to measure $\rho_{\max }$ and calculate $F_{\text {th }}$ for different Ns.

Applying equations (1) and (2) it was possible determine ablation threshold fluence as function of $\mathrm{N}$ and the incubation behavior of the material. Figure 2 shows the ratio of $F_{\text {th }}$ $\mathrm{x} N$ obtained for these traces. The best-fit curve obtained define the incubation effect for steel DIN $16 \mathrm{MnCr} 5$.

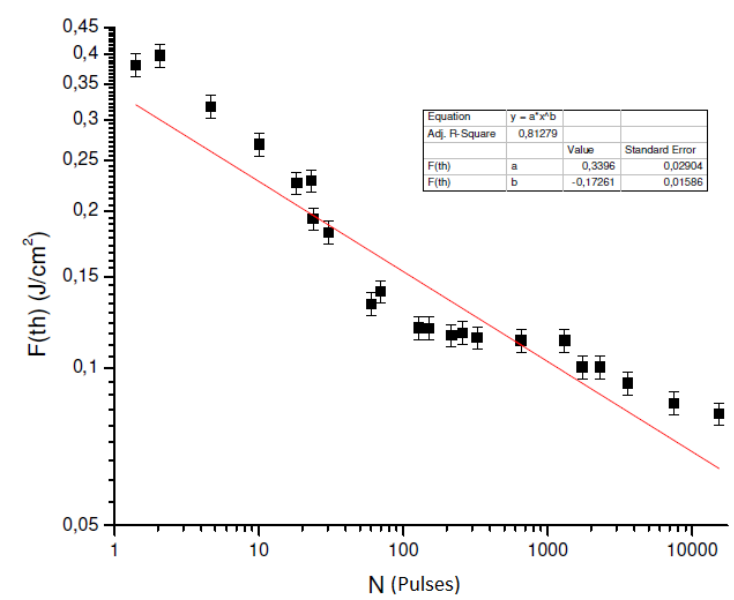

Fig. 2 Dependence of ablation threshold fluence $F_{\text {th }}$ on the pulse overlapping $\mathrm{N}$ obtained by the $\mathrm{D}$-Scan method to DIN $16 \mathrm{MnCr} 5$ steel

The constant downward line is typical for metallic materials, showing that there is a strong reduction of $F_{\text {th }}$ with increasing $\mathrm{N}$. This means that, in machining, even starting the process with fluence close to the threshold it may become well above the threshold in the course of the process.

This effect is known as the incubation effect, and for metals, the relationship between $F_{t h}$ and $\mathrm{N}$ is given by the expression below [7]:

$$
F_{t h, N}=F_{t h, 1} N^{(S-1)}
$$

$\mathrm{F}_{\text {th } 1}$ and $\mathrm{F}_{\text {thN }}$ are the ablation thresholds for 1 and $\mathrm{N}$ overlapping pulses respectively, and $\mathrm{S}$ is the incubation parameter. $\mathrm{S}$ ranges from 0 to 1 , and the closer to zero, the greater the effect. In the case of the plot of figure $2, \mathrm{~S}=0.81$, and $\mathrm{F}_{\text {th }}$ was reduced from $\sim 0.4 \mathrm{~J} / \mathrm{cm}^{2}$ for one shot to $\sim 0.1 \mathrm{~J} / \mathrm{cm}^{2}$ for 1,000 overlapped shots.

The knowledge of this behavior, and the relationship between $F_{\text {thN }} \times N$, is useful to identify regions where the appearance of thermal effects is prevented. Thus, the use of fluences near the ablation threshold can, in principle, reduces or avoid the formation of molten and resolidified material (burrs), as well as a possible phase transformation in the heat affected zone (HAZ).

D-Scan traces were also used to help in choosing the process parameters for the production of the dimples. Thus, dimensional and morphological characteristics of the traces were observed, such as uniformity, symmetry, absence of resolidified material and, among others, signs that could indicate presence of thermal effects, absence or minimal presence of ripples, profile and dimensional characteristics, such as width and depth. One region with these features can be observed in Fig. 3. The process parameters in this case are the starting point for the production of the specified dimples. 
Using the straighter region of the trace as a reference (focus on the surface), the location of the region of interest was find at a distance of $1.25 \mathrm{~mm}$ to the left. As longitudinal and transversal speeds are the same, at this position the focal point is located $1.25 \mathrm{~mm}$ above the surface. Here the diameter of the beam on the surface was calculated and a fluence of $100 \mathrm{~mJ} / \mathrm{cm}^{2}$ and overlap of $\mathrm{N}=320$ pulses was obtained. At this point the trace is $104.5 \mu \mathrm{m}$ wide and $46.8 \mu \mathrm{m}$ deep. The fluence here is much greater than that of the threshold for this number of pulses, certainly leading to the production of molten material. Due to this very high fluence, this liquid material is almost completely expelled out of the machined region by processes of phase explosion and spallation [14].
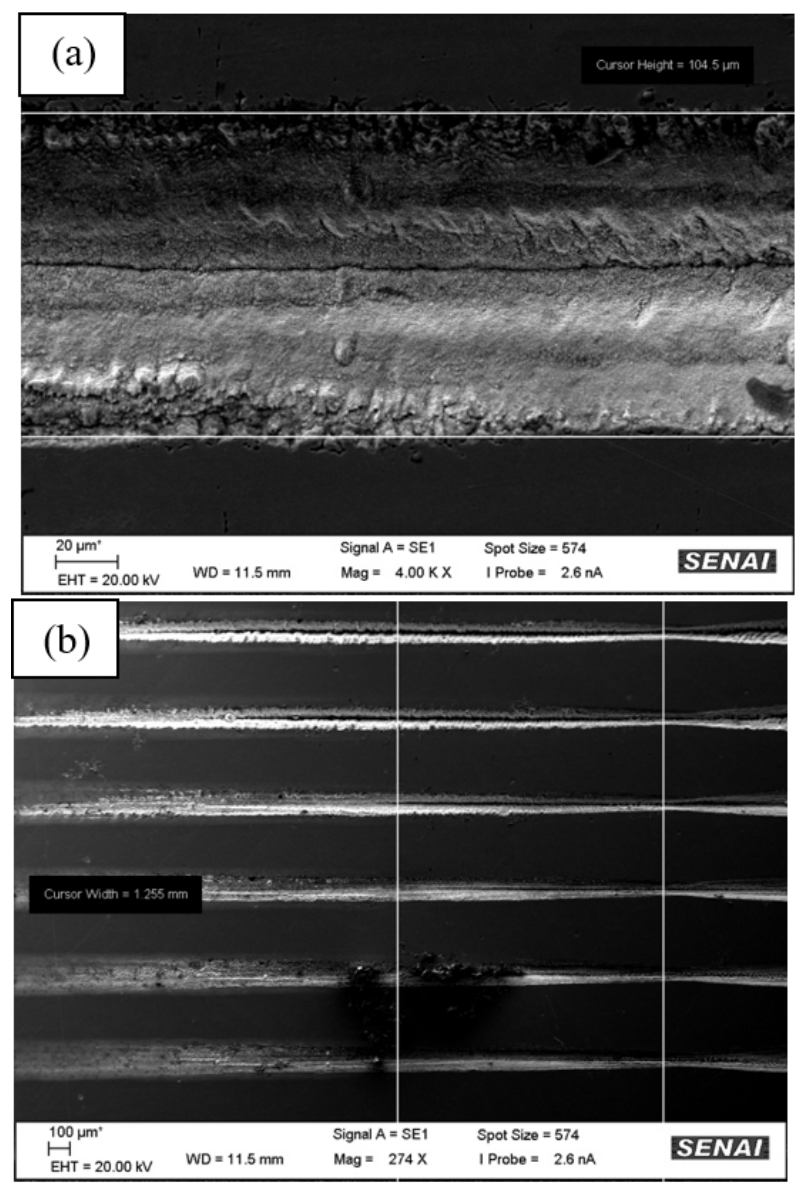

Fig. 3 Image obtained by SEM of trace with $N=320$. (a): region located to the left of the center of the trace. (b): Measure of the distance between the center of the trace and the region of interest

The use of these process conditions still presents a drawback as can be seen in Fig. 3a, that is a deep groove in the center of the trace, undesired for the process. Fig. 4 shows a $3 \mathrm{D}$ figure of the region analyzed in Fig. 3, obtained from the confocal microscope, and a cross-sectional profile. In addition, the "V" shape of the groove is not suitable for what is intended for a dimple.

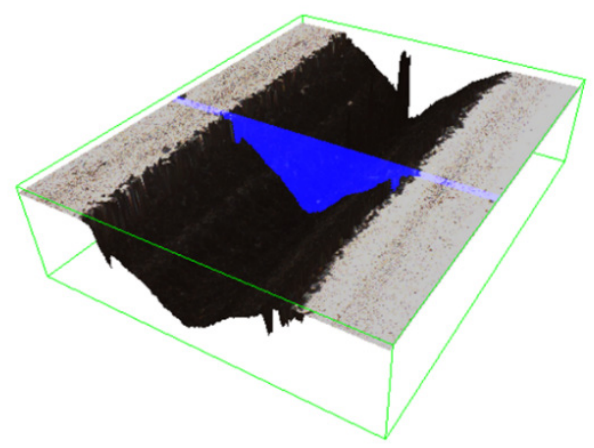

Fig. 4 3D profile of the region of interest seen by confocal microscope.

The deepest region at the center of the trace is a result of the higher peak intensity of the Gaussian spatial energy distribution, reinforced by the incubation effect. To circumvent this effect, a donut-shaped beam was created by inserting an iris into the beam path before the focusing lens. Here the iris diameter was six times smaller than the diameter of the laser beam. The beam profile formed behind the focusing lens is shown in figure 5 .

In focus, this donut-shaped beam also features an intense peak in the center with rings of low intensity at the edges. Far from focus, the intensity distribution is that of a ring with zero intensity in the center. None of these positions is suitable for the production of a cavity with semi-spherical profile and an intermediate position must be sought to produce such a structure.

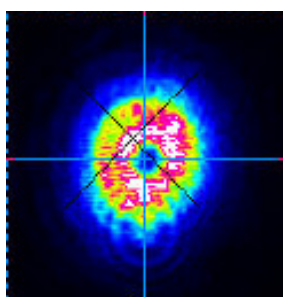

Fig. 5 Profile of the donut shaped laser beam with reduction of intensity in the center.

The search for the most adequate structure was made by varying the position of the focal point, the pulse energy and the number of overlapping pulses. Based on the analysis of the D-Scan traces, it was decided to vary the focus position by steps of $100 \mu \mathrm{m}$ down and up from the surface. The energies used were $5.0 \mu \mathrm{J}$ and $9.3 \mu \mathrm{J}$, with four different Ns: $100,200,400$ and 1000, and beam with circular polarization.

Through this experiments it was possible to observe dimples with more uniform surfaces and with a semi-spherical geometric characteristic. It was also observed that even with the variation of the number of pulses and energy, the diameter of the dimples remained very close to the value of $50 \mu \mathrm{m}$. The depth varied according to the number of laser shots. The best result was observed to focus spot positioned $200 \mu \mathrm{m}$ above surface and energy of $5.0 \mu \mathrm{J}$, where the deepness presented a linear variation of approximately $0.024 \mu \mathrm{m}$ per pulse. The diameter of the dimples remained in the range of 50 to $52 \mu \mathrm{m}$. Fig. 6 shows two graphs with the results correlating the variation of the depth in relation to the number of pulses and the variation of the focus position. 
Figure 8 shows a comparison between the dimples made with different overlapping number of pulses and focus position $200 \mu \mathrm{m}$ above surface.
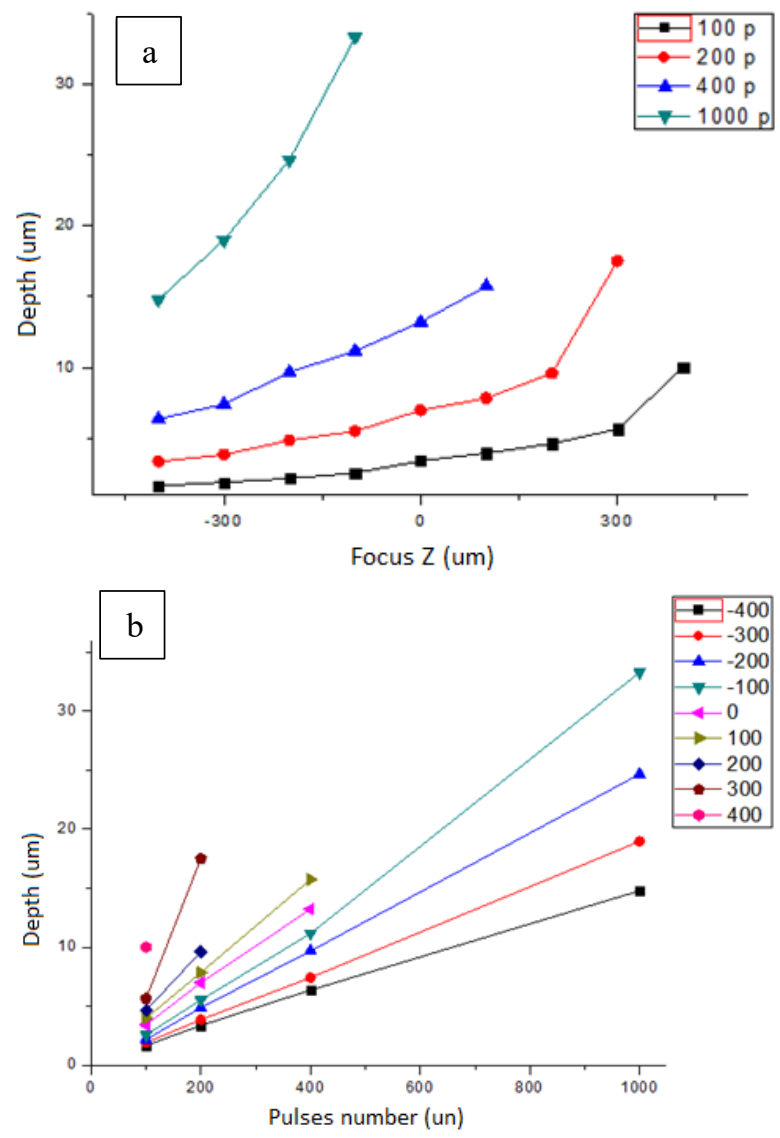

Fig. 6 Depth variation: (a): relative to the position of the focus (b): in relation to the number of pulses.

A particular microstructure was chosen for the tribological tests. Based on $[11,13]$ and other empirical means it was chosen a dimple that presented the relation between diameter and depth in the order of 10/1 and two texture density of $7 \%$ and $11 \%$ of the surface, according figure 7 .

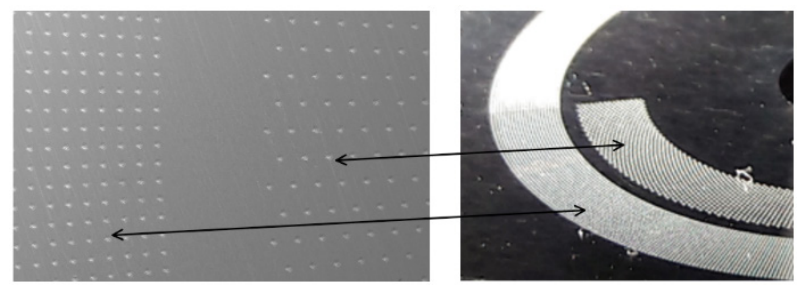

Fig. 7 textured regions covering $11 \%$ (left) and $7 \%$ (right)

The density of $7 \%$ was found to be inadequate for the test conditions used here, i.e. sphere-on-disk. In this case, the density of dimples is very low presenting distance between the cavities much larger than the contact area.

A tribological disc-sphere test was performed with lubricated contact according to the parameters already described, and with no variation of any of the parameters; the test was repeated three times with variation in the radius of the sample and respective variation of rotation of the equipment to maintain the sliding velocity initially proposed. In addition, tests were also performed on the same disk in a non-texturized zone. To compare the results, the corresponding values of the friction force and the coefficient of friction were recorded.
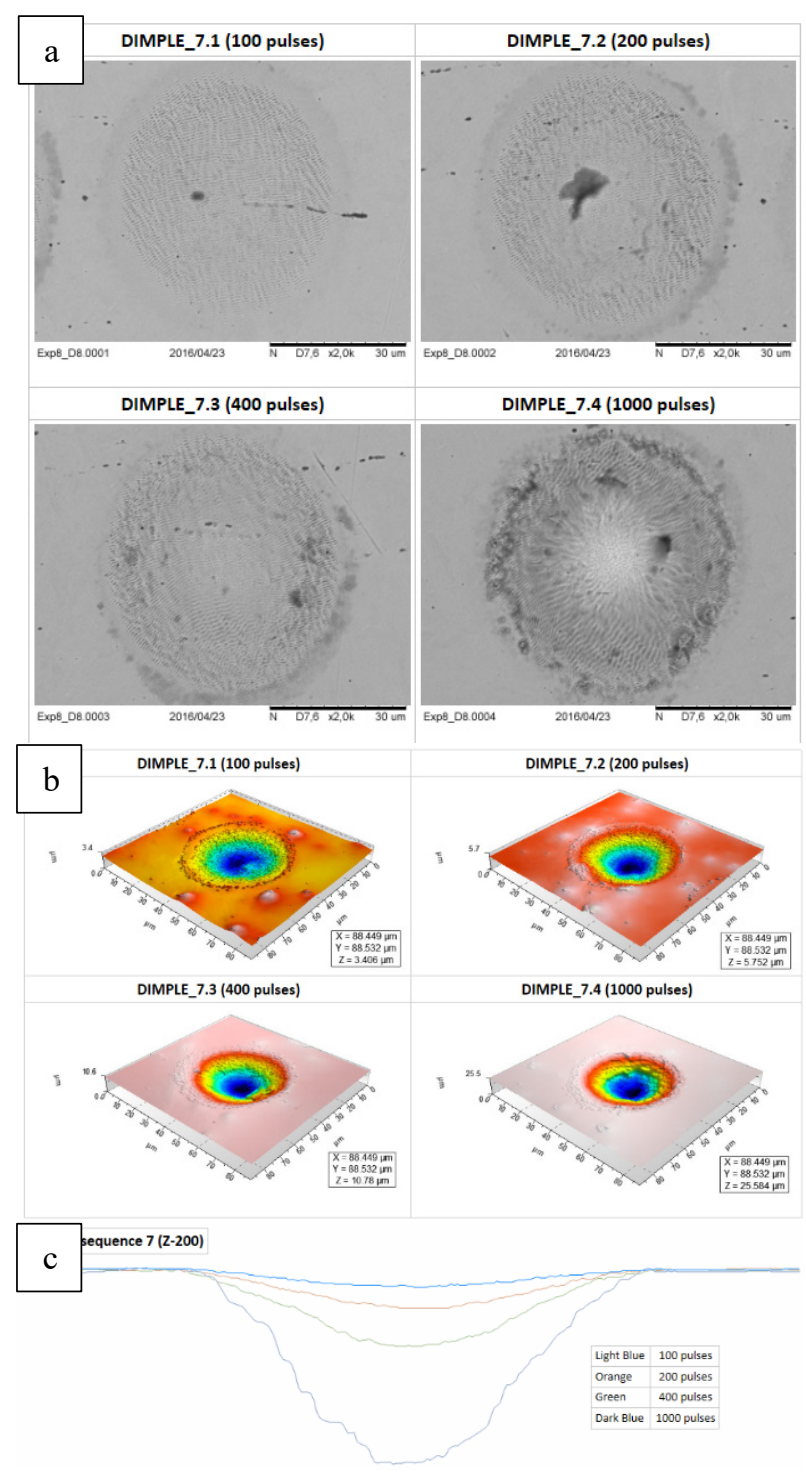

Fig. 8 (a) Images obtained by SEM (b) 3D surfaces obtained by WLI (White Light Interferometry) (c) 2D profiles obtained by WLI

In the region without texture, an average value of $2.94 \mathrm{~N}$ was found for friction force and 0.105 for the coefficient of friction. In the region with $11 \%$ of texture density the measured friction force was $2.61 \mathrm{~N}$ and the coefficient of friction was 0.085 . The values were obtained by means of the average of three tests, which, individually, presented similar values. The average reduction of the friction force in the textured zone was $11 \%$ and the reduction of the coefficient of friction reached $18 \%$. Images obtained by SEM (Scanning Electron Microscopy), can be observed in figure 9. 


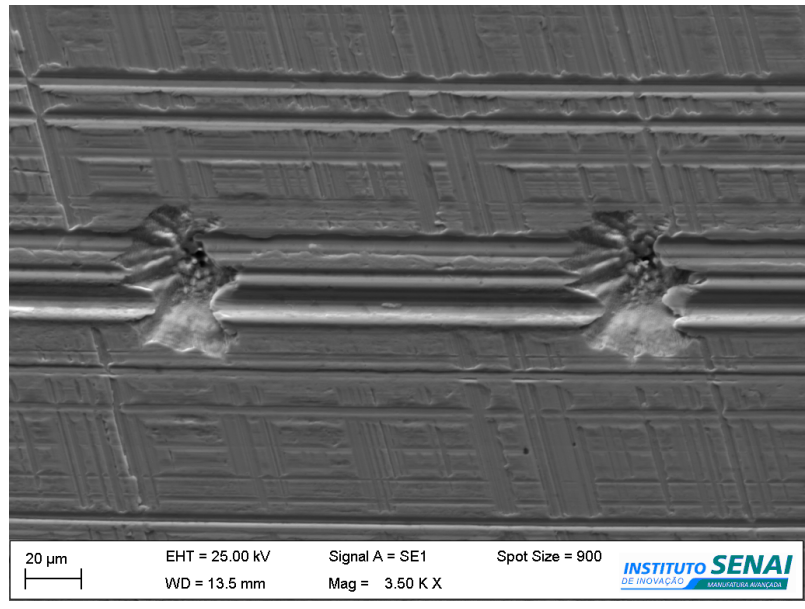

Fig. 9 Image obtained by SEM of tested region for $11 \%$ textured zone.

The area of contact between the sphere and the surface of the disk is given by the deformation caused by the normal force, in this case $0.025 \mathrm{~mm}^{2}$. The wear of the textured region, although it had lower coefficient of friction, presented the creation of a groove of considerable depth. This event is attributed to the increase of the contact pressure in the region of the dimple, resulting in the deformation of the dimples (Figure 8) and increasing wear. It is worth noting that this is only valid for this type of contact (with the sphere) and that for other conditions wear must be considerably reduced.

\section{Conclusion}

The D-Scan technique is useful not only to determine the relation $\mathrm{F}_{\text {th }} \mathrm{x} \mathrm{N}$ but also to find regions with specific morphologies as a function of the process parameters.

The control of the focal position of a donut-shaped beam can lead to the production of a microstructure in the form of a semi-sphere.

The control of the energy and the number of overlapping pulses leads to a control of the diameter and depth of the structure. The depression in the center of the intensity profile prevents the formation of a deep channel at the bottom of the dimples. The obtained dimples with the presented parameters, has shown good geometrical characteristics. The use of fluence well above the threshold leads to the expulsion of molten material away from the machined region leaving the surface clean, without resolidified material and burrs avoiding the necessity of rework of the textured surface.

The tribological tests showed that although the textured surface presents a considerable reduction of the coefficient of friction in boundary conditions of the contact, the reduction of the area of contact caused by the presence of the micro cavities, results in a considerable increase of the contact pressure, causing a premature wear the material. In this way, the use of laser texturing would be interesting in applications where the contact area has a much larger proportion than the area of the micro cavities, making the contact pressure increase relatively small or even negligible. This is the case foreseen for piston pins used in automotive engines.

This type and density of microstructure, can therefore, lead to important gains in terms of components lifetime reducing energy to overcome losses due to friction.

Acknowledgments: The authors are grateful for the technical and financial support of .FAPESP Proj. 2013/26113-6; CNPq Proj. 307063/2016-0 and SENAI Innovation Institute Advanced Manufacturing and Microproduction.

\section{References}

[1] R. E. Samad, L. M. Machado, N. D. Vieira Jr. and W. de Rossi: "Laser Pulses - Theory, Technology and Applications - Ultrashort Laser Pulses Machining" ed. by I. Peshko, (InTech, Rijeka, 2012) Chap. 5, p. 143.

[2] I. Etsion: Tribol. Lett., 17, (2004) 733.

[3] I. Etsion and E. Sher: Tribol. Int., 42, (2009) 542.

[4] I. Etsion: Proc. ASME 7th Biennial Conference on Engineering Systems Design and Analysis. American Society of Mechanical Engineers, Manchester, (2004) p. 585 .

[5] G. Boidi, I. S. Tertuliano, F. J. Profito, W. de Rossi and I. F. Machado: Tribol. Int., (2019) p. 1.

[6] M. Bertolete, P. A. Barbosa, A. R. Machado, R. E. Samad, N. D. Vieira Jr. And R. Vilar: Int. J. Adv. Manuf. Techol., 98, (2018) p. 2653.

[7] Y. Jee, M. E. Becker and R. Walser: J. Opt. Soc. Am. B., 5, (1998) 648-659.

[8] J. Lopez, M. Faucon, R. Devillard, Y. Zaouter, C. Honninger, E. Mottay and R. Kling: J. Laser Micro/Nanoengin., 10, (2015) 1.

[9] A. Horn, U. Klug, J. Dusing, J. G. Moreno, V. Schutz, O. Suttmann and L. Overmeyer: "Ultrashort Pulse Laser Technology: Laser Sources and Applications - Micromachining" ed by S. Nolte, F. Schrempel, F. Dausinger (Springer, Jena, 2016) p. 155

[10] K. Holmberg, P. Andersson and A. Erdemir: Tribol. Int., 47 (2012) 221.

[11]F. J. Profito: "One-dimensional modeling of the mixed lubrication regime applied to textured surfaces". (masters dissertation) - University of São Paulo. São Paulo, (2010) p 34.

[12]L. M. Machado, R. E. Samad, W. de Rossi and N. D. Vieira Jr: Opt. Express, 20, (2012) 4114.

[13] A.Vieira: "Analysis and study of parameters for laser texturization with ultrashort pulses to improve the tribological properties of motor components". (masters dissertation) - University of São Paulo. São Paulo (2012).

[14]] E. G. Gamaly: Phys. Rep., 508, (2011) 91.

(Received: May 14, 2019, Accepted: August 13, 2019) 